\begin{tabular}{ccc}
\hline International Journal of Engineering \&Technology, $7(1.8)(2018)$ 144-147 \\
SPC \\
Website www.sciencepubco.com/index.php/IJET \\
Research paper
\end{tabular}

\title{
WT-ANN based fault discrimination for transmission system
}

\author{
P. Venkata Lakshmi, P. N. S. Poojitha, Y. Srinivasrao
}

\begin{abstract}
${ }^{1}$ Electrical\&Electronics Engineering, Koneru Lakshmaiah Education Foundation,GreenFields, Vaddeswaram
${ }^{2}$ Electrical\&Electronics Engineering, Koneru Lakshmaiah Education Foundation,GreenFields, Vaddeswaram

3 Electrical\&Electronics Engineering, Koneru Lakshmaiah Education Foundation,GreenFields, Vaddeswaram

*Corresponding author E-mail:lakshmiv904@gmail.com
\end{abstract}

\begin{abstract}
Protection of transmission line is a complex in power system as the majority of the faults in power system are transmission faults. A proper protection is needed for transmission line for continuous power supply. To provide a strong as well as an efficient protection scheme, in this paper we are using wavelet technique and artificial neural network. By using these mentioned two techniques we can detect the faults in transmission line and also, we can classify the detected faults. Wavelet transform has strong mathematical, very fast and accurate tools for brief signal inside the transmission lines and synthetic neural network can make a unique between measured sign and associated signal that has different pattern.
\end{abstract}

Keywords: Wavelet, Neural Network, Fault, Transmission Line

\section{Introduction}

\subsection{About Transmission System Faults}

In recent years, with increase of the electricity, the research of an automated , dependable method of safety device has aroused big attention. Transmission traces being vital part in a strength gadget and the faults will create interruption and decrase the security of the complete machine. The electric power distribution networks are continuously subjected to occasions that generate interruptions within the supply of power to the end customers, which may be resulting from a variety of factors consisting of awful weather situations, contact between trees and accomplishing wires, system failure, unpredictable injuries and plenty of more. These interruptions decrease the first-class indices of these circuits, inflicting penalties for the businesses because of such conditions. Therefore, its miles required strategies and strategies to improve the manner of fault place in distribution networks. When a surprising exchange, consisting of a fault or a disturbance occurs on a transmission line, a touring wave could be generated, and it will propagate at nearly the speed of light. It is a considerable amount of work to characterize and finding the transients by way of most effective the use of the authentic statistics.

The important task in power system is to classify the faults in transmission line. The protective relay has a selector module to classify the sort of fault which taken place, additionally categorize "normal country". To identify fault is consequently vitally critical if you want to keep away from both wrong section tripping or pointless three-phase tripping. Important need of section selectors is more pace operation as the choice technique have to be finished within the immediately submit-fault time . Traditional phase choice schemes be afflicted by a few drawbacks because of complication device, unawareness of its parameters, effect of remote-quit infeed, fault resistance, mutual-coupling from adjoining parallel strains, . To adapt dynamically the system has no capacity, and it is impossible to make correct selection if the alets are not proper. To detect and category the fault is a very difficult mission. There are various methods like travelling waves, adaptive filtering, fuzzy common sense, artificial neural networks, and the fusion of various synthetic intelligence techniques. On these methods different attempts were made to find fault. Several researchers proposed distinct techniques to identify transmission line fault like distinctive kinds of neural networks and their combination with desparate transformations, inclusive of wavelets and hyperbolics. The neural-network methods had been quite a success in determining the appropriate fault kind, principle drawback of neural-network is specifically below huge version of working conditions. Another drawback of artificial neural network primary algorithms is that the schooling is unconverged at some ofthe instances, random selection and can turn out to be in a local minimum

\subsection{Artificial Neural networks (ANN)}

The computing system of artificial intelligence neural network is simple because of interconnected elements. The interconnected elements has different weights which can be adjusted during the process of training. In the field of statitics,signal processing artificial neural network can be used. 


\section{Related Work}

Some of the reasearchers proposed a technique to identify and classify the fault. The approach makes use of the samples of modern-day and current extracted from the fault . The brief power characteristic from those sampled alerts can be extracted though wavelet transformation. This characteristic vector then acts as enters SVM for training., The techniquewhich is proposedturned into also loose from many problems which might be faced via traditional neural networks processes, which include generalization Technique may be prolonged to locate flaw and type of flaw on a chain transmissionline lines, but few functions are needed for SVM training.

Fuzzy logic is a method to identify and classify the fault on transmissionline determined to be very adequate under unique flawsituations .The system operation is rapid, reliable, and at ease. The logic is simple because it calls for just a few guideline. The results display that proposed techniques is easy, stable and comfy.

A unique approach is used the defective stages in a transmission line. A recommended approach is primarily based on dimension of section currents and speedy estimation of phasor additives in rather quick statistics window. The important choice technique makes use of relations between present day magnitudes for exceptional feasible flaw loops. The retive magnitude of neutral and phase currents are used to differentiate the grounded and ungrounded flaw. The proposed approach is impartial of the gadget configuration, and the energy system working conditions during faults. The technique is likewise computationally efficient. This method requires no programming device structured parameters, including 0 -series impedances, thereby presenting greater flexibility to users.

Pragati Chawardol and Heena Shaikh provided a noncommunication protection approach for transmission traces. The Threshold subsystem technique became hired to divide the faults in accordance with fault currents components decomposed by using the wavelet rework. Software of wavelet transform to virtual distance safety of transmission line changed into presented on this version. Use of wavelet rework offers the capability to locate faults along with excessive impedance fault. Wavelet based distance safety scheme has been examined the usage of MATLAB model 2010 laptop simulation model. Proposed work successful to diagnosed main faults in less than half cycle after fault inception; present paintings studied some crucial elements which impact the operation relay. Efficiency of this threshold device is up to ninety two $\%$ calculated from extraordinary regular and unusual 246 cases at specific fault region. Proposed approach extended for protection of double circuit transmission line and multi terminal transmission strains. Proposed approach prolonged with aggregate of wavelet rework and neural network for electricity nice observation and fault identification.

\section{Methodology}

There are extraordinary methods that could help to discover the place of fault, additionally to locate the fault types. Here we are able to use wavelet transform as a one of the exceptional tools to discover the location of the fault which is ship an signal at some point of the transmission strains and with the measuring the time of returning that sign, locate the area which the fault is passed off. We can use another tools to find the forms of fault that's neural network.

\subsection{Terminology}

\section{Wavelet Transform:}

Wave let transform is same as fourier transformation. The major difference between these techniques is in wavelet transformation frequency components with timelocalisation of the given signal Wave let analysis has been efficient technique where signal has been analysed by transistors or discontinues. In wave let transform the analysing functions are wavelets.The signal is analysed by waveletfunction known as mother wavelet .The mother wavelet depends on type function.

\section{Artificial Neural Network:}

Artificial Neural Networks (ANN) can exhibit traits which includes sample affiliation. Different type of examples are used to learn artificial neural network. This study makes to acquire more knowledge about arterial neural networks

\subsection{Trnsmission line fault detection and classification}

Fault currents are taken from the system and to these signals wavelets are applied .The detetction of fault is carried out through waveletcoffecients .By using wavelet coefficients the energies are calculated.These energies are used to detect and classify the flaw.

\section{A. Fault Detection Using Discrete Wavelet Transform (DWT)}

The transmission line has high voltage, fault is one of the reason for voltage sag. When a flaw occurs there will be more voltage drop.The circuit breaker deenergizes the line as flaw is occurred.The frequency and time are required to analyse the flaw .The modern waveforms are analysed with respect to time and frequency, in DWT the first decomposition is also used to detect the flaw.In DWT decomposition of first stage consists of highest frequency addictives.DWT has an advantage over continuous wavetransform the computional burden can be decreased.

\section{B. Fault Classification Using Artificial Neural Network (ANN)}

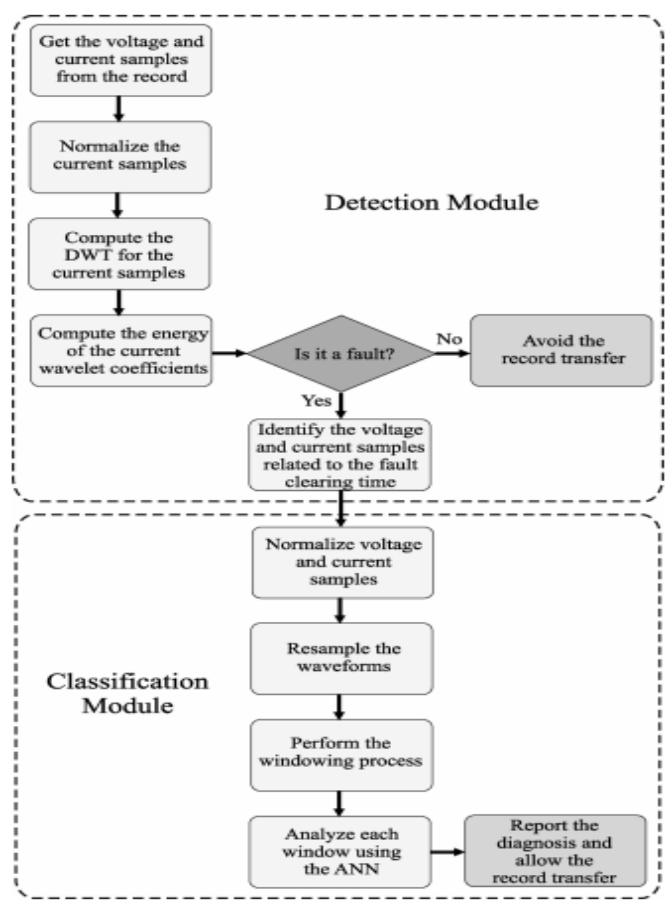

Fig1. Fault detection and Classification in Transmission line

Order to categorise faults, a Multilayer Perceptron (MLP) network is used, which ought to gain knowledge of before implementing the proposed approach. In this manner, the learning database ought to include a incredible style flaw situations to improve the ANN's training. Regardless, a ramification of flaw isn't discovered with actual flaw records. Therefore it is vital to use simulated fault records to perform the neuralnetwork mastering. The usage of this technique, the ANN can analyse effectively assumed and actual flaws.The artificilintelligence neural network output gives the type of flaw similar to the enter sample. 


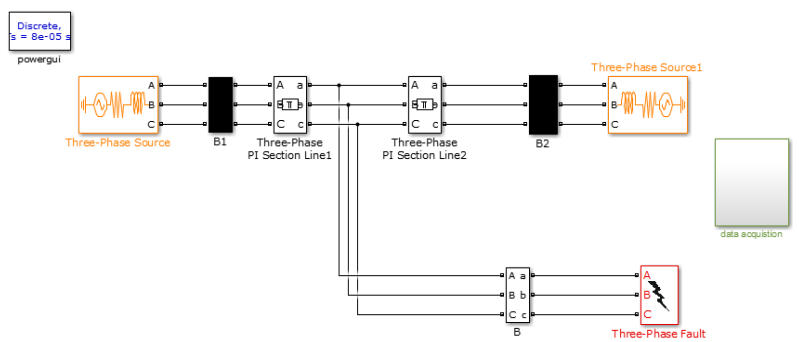

Fig (1):consider transmission line which is length $300 \mathrm{Km}$ which has two sources on bothside with voltage 400volts .

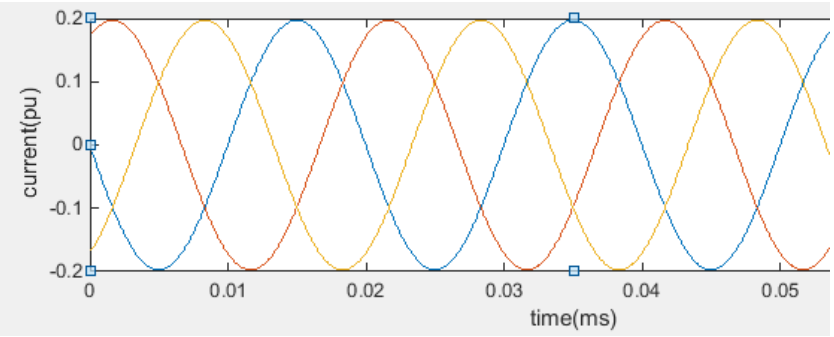

Fig(2): under normal condition i.e when there is no fault the currents are in phase, Phase currents for Healthy system.

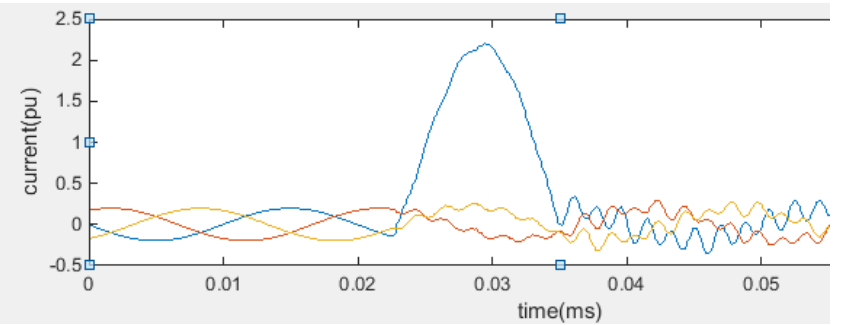

Fig(3): when there is flaw in phase A we observe that the current in phase $\mathrm{A}$ is more when compared to other phases.

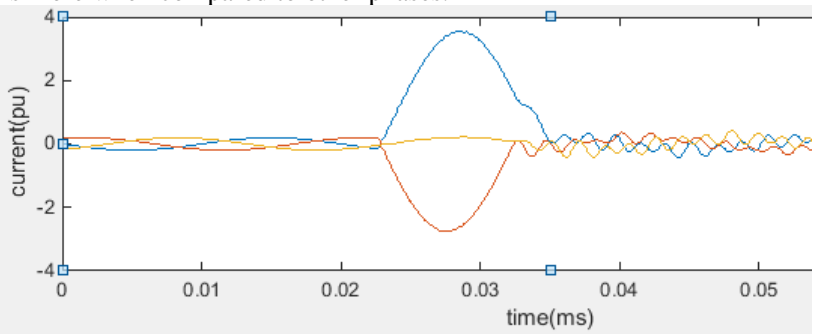

Fig(4): when there is flaw between A and B phase the currents are more when compared to $\mathrm{c}$ phase

After creating the fault the wavelet decomposition has been applied to the current waveforms.Daubchies wavelet 'db4'is used and using wavelet decomposition .The sampling frequency is used $10 \mathrm{khz}$. The frequency of the system is $50 \mathrm{~Hz}$ and number of samples is 200.Using Wavelet decomposition the energy packetsare produced.The energies are given as input to neural networks and type of fault and distance are given as output.The training is performed using neural network.
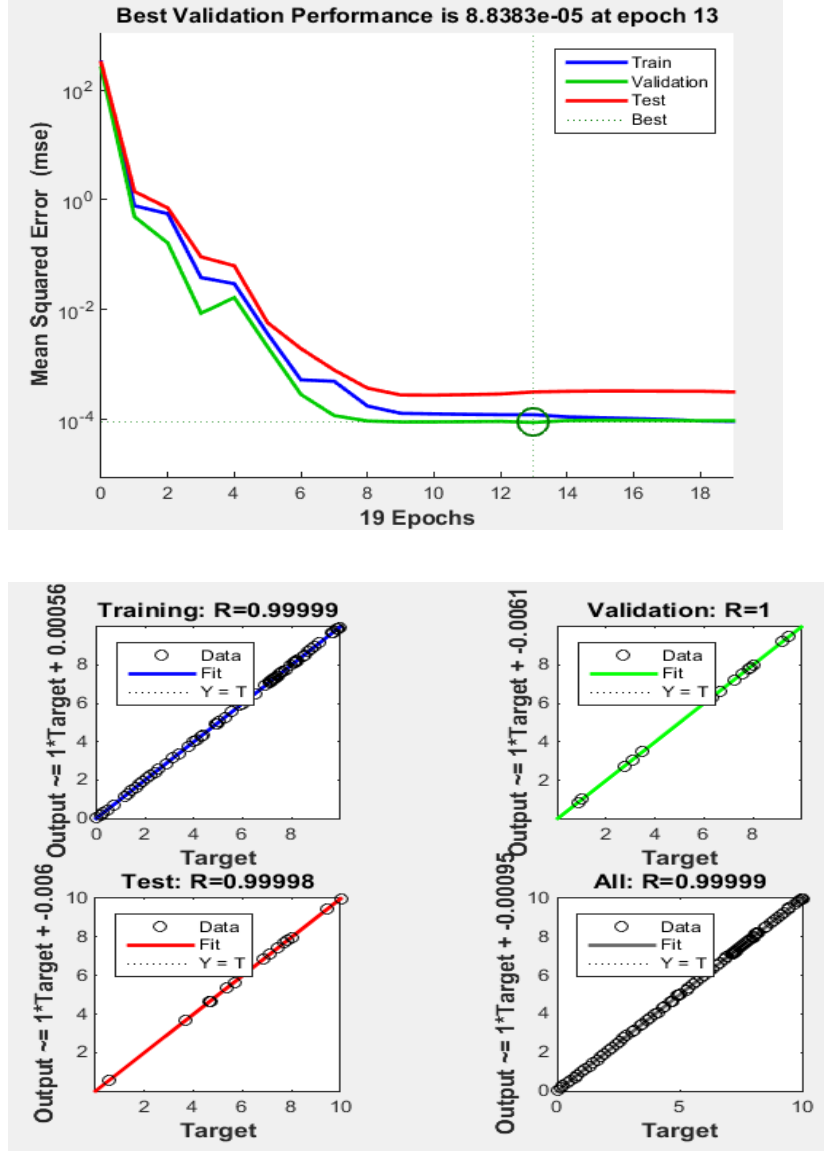

Fig(6):

The figures 5 and 6 are about the type of fault ,the inputs are given as energies, fault inception angle, fault resistance and distance ,outputs are type of fault are given to the nerual networks for training. We can say that the training is done upto $99 \%$ from this we can say that the system can identify which type of fault.

\section{Conclusion}

In this paper we implemented wavelet technique along with artificial neural network to detect as well classify the faults in transmission line which are caused in transmission systems. The implemented system can have worked on finding different types of fault in transmission lines with the help of two different materials. DWT is used to decompose cutting-edge indicators into different frequency bands and based on it the character of fault is diagnosed Neural network is used to discover the one-of-a-kind styles of fault while wavelet rework is used to find the area of the fault.

\section{References}

[1] Tang Y, Wang HF, Aggarwal RK et al., -Fault indicators in transmission and distribution systemsll, Proceedings of International conference on Electric Utility Deregulation and Restructuring and Power Technologies - DRPT, 2000, pp. 238-243.

[2] Youssef OA. Fault classification based on wavelet transforms. In Transmission and Distribution Conference and Exposition, 2001 IEEE/PES 2001; 1: pp. 531-536

[3] Manohar singh, Dr. B.K Panigrahi and Dr. R. P. Maheshwari, "Transmission Line Fault Detection and Classification", PROCEEDINGS OF ICETECT 2011

[4] Shuma Adhikari, Nidul Sinha and Thingam Dorendrajit, "Fuzzy logic based on-line fault detection and classification in transmission line", SpringerPlus (2016) 5:1002 DOI 10.1186/s40064016-2669-4

[5] R. K. Aggarwal, Q. Y. Xuan, A. T. Johns, R. W. Dunn and A.Bennett, "A novel fault classification technique for doublecir- 
cuit lines based on a combined unsupervised/supervised neural network," IEEE Transactions on Power Delivery, Vol. 14, No. 4, pp. 1250-1256, October 1999

[6] Pragati Chawardol and Heena Shaikh, "Wavelet Entropy Based Transmission Line Protection", Volume: 04 Issue: 04 | Apr -2017

[7] Prasad A, Edward JB, Roy CS, Divyansh G, Kumar A. Classification of Faults in Power Transmission Lines using Fuzzy-Logic Technique. Indian Journal of Science and Technology. 2015; 8(30):1-6.

[8] K. M. Silva, B. A. Souza and N. S. D. Brito, "Fault Detection and Classification in Transmission Lines Based on Wavelet Transform and ANN", IEEE Transactions on Power Delivery, Vol. 21, No 4, OCTOBER 2006

[9] T. Dausten and B. Kulieke, "Neural network approach to fault classification for high speed protective relaying," IEEE Trans. Power Del., vol. 10, no. 2, pp. 1002-1009, Apr. 1995.

[10] W. A. Wilkinson and M. D. Cox, "Discrete wavelet analysis of power system transients," IEEE Trans. Power Syst., vol. 11, no. 4, pp. 2038-2044, Nov. 1996 